\title{
Dutasteride Treatment Over 2 Years Delays Prostate-specific Antigen Progression in Patients with Biochemical Failure After Radical Therapy for Prostate Cancer: Results from the Randomised, Placebo-controlled Avodart After Radical Therapy for Prostate Cancer Study (ARTS)
}

\author{
Fritz Schröder $^{a, *}$, Chris Bangma ${ }^{a}$, Javier C. Angulo ${ }^{b}$, Antonio Alcaraz $^{c}$, Marc Colombel $^{d}$, \\ Tom McNicholas ${ }^{e}$, Teuvo L. Tammela ${ }^{f}$, Indrani Nandy ${ }^{g}$, Ramiro Castro ${ }^{h}$ \\ ${ }^{a}$ Erasmus Medical Centre, Rotterdam, The Netherlands; ${ }^{b}$ Hospital Universitario de Getafe, Madrid, Spain; ${ }^{\mathrm{c}}$ Urology Department, Hospital Clinic, Barcelona, \\ Spain; ' Service d'Urologie et Chirugie de la Transplantation, Université Lyon 1, Lyon, France; ${ }^{\mathrm{e}}$ South Bedfordshire E Hertfordshire Urological Cancer Centre, \\ Stevenage, UK; ${ }^{\mathrm{f}}$ Tampere University Hospital, Tampere, Finland; ${ }^{\mathrm{g}}$ GlaxoSmithKline, Research Triangle Park, NC, USA; ${ }^{\mathrm{h}}$ GlaxoSmithKline Research and \\ Development, King of Prussia, PA, USA
}

\section{Article info}

Article history:

Accepted November 4, 2012

Published online ahead of print on November 12, 2012

\section{Keywords:}

$5 \alpha$-reductase inhibitor

Biochemical failure

Disease progression

Prostate cancer

\begin{abstract}
Background: Rising prostate-specific antigen (PSA) levels after radical therapy are indicative of recurrent or residual prostate cancer $(\mathrm{PCa})$. This biochemical recurrence typically predates clinically detectable metastatic disease by several years. Management of patients with biochemical recurrence is controversial.

Objective: To assess the effect of dutasteride on progression of PCa in patients with biochemical failure after radical therapy.

Design, setting, and participants: Randomised, double-blind, placebo-controlled trial in 294 men from 64 centres across 9 European countries.

Intervention: The $5 \alpha$-reductase inhibitor, dutasteride.

Outcome measurements and statistical analysis: The primary end point was time to PSA doubling from start of randomised treatment, analysed by log-rank test stratified by previous therapy and investigative-site cluster. Secondary end points included time to disease progression and the proportion of subjects with disease progression.

Results and limitations: Of the 294 subjects randomised (147 in each treatment group), 187 (64\%) completed 24 mo of treatment and 107 discontinued treatment prematurely (71 [48\%] of the placebo group, 36 [24\%] of the dutasteride group). Dutasteride significantly delayed the time to PSA doubling compared with placebo after 24 mo of treatment $(p<0.001)$; the relative risk (RR) reduction was $66.1 \%$ (95\% confidence interval [CI], 50.35-76.90) for the overall study period. Dutasteride also significantly delayed disease progression (which included PSA- and non-PSA-related outcomes) compared with placebo $(p<0.001)$; the overall RR reduction in favour of dutasteride was $59 \%$ (95\% CI, 32.53-75.09). The incidence of adverse events (AEs), serious AEs, and AEs leading to study withdrawal were similar between the treatment groups. A limitation was that investigators were not blinded to PSA levels during the study. Conclusions: Dutasteride delayed the biochemical progression of PCa in patients with biochemical failure after radical therapy for clinically localised disease. The safety and tolerability of dutasteride were generally consistent with previous experience. Clinical trial registry: ClinicalTrials.gov, NCT00558363.
\end{abstract}

(C) 2012 European Association of Urology. Published by Elsevier B.V. All rights reserved. E-mail addresses: secr.schroder@erasmusmc.nl, f.h.schroder@hetnet.nl (F. Schröder). 


\section{Introduction}

Increasing adoption of prostate-specific antigen (PSA) testing has led to a migration in the stage and grade of prostate cancer $(\mathrm{PCa})$ at diagnosis such that most affected men present with localised disease [1]. While most patients with clinically localised disease undergo radical prostatectomy (RP) or radiotherapy (RT), local or distant recurrences develop in up to half of intermediate- or high-risk patients within $10 \mathrm{yr}$ [2].

Elevated or rising PSA level after radical therapy is indicative of recurrent or residual PCa. This biochemical recurrence typically predates clinically detectable metastatic disease by several years [3]. European Association of Urology (EAU) guidelines recommend watchful waiting with possible delayed androgen-deprivation therapy (ADT), or salvage RT, in patients with presumed local failure after $\mathrm{RP}$ [2]. A similar approach is recommended for patients with presumed local failure after RT. Nevertheless, the management of these patients is complex and controversial [4].

The Avodart After Radical Therapy for Prostate Cancer Study (ARTS) assessed the effect of dutasteride (Avodart; GlaxoSmithKline plc, Brentford, Middlesex, UK) on PCa progression in patients with biochemical failure after radical therapy [4]. In this paper, we report the key efficacy and safety findings from the study.

\section{Patients and methods}

This was a 2-yr, multicentre, randomised, double-blind, placebocontrolled trial. Eligible men were $<85 \mathrm{yr}$ of age with asymptomatic PSA failure following radical therapy with curative intent for clinically localised PCa. Definitions of PSA failure were based on the recommendations from the EAU guidelines on PCa (for subjects treated with RP) or from the Radiation Therapy Oncology Group-American Society for Therapeutic Radiology and Oncology 2005 Consensus Conference (for subjects treated with RT) [2,5]. Additional entry criteria included serum PSA levels of $2-20 \mathrm{ng} / \mathrm{ml}$ for men treated with primary RT or $0.4-10 \mathrm{ng} / \mathrm{ml}$ for men treated with RP with or without salvage RT; PSA doubling time (PSADT) $>3$ mo and $\leq 24$ mo; clinical stage T1-T3a N0 M0; nonmetastatic PCa as confirmed on negative bone scan within 6 mo prior to randomisation; no evidence of local recurrence in RP or salvage RT subjects; expected survival $\geq 2$ yr; and Eastern Cooperative Oncology Group performance status 0, 1, or 2. Sixty-four centres in nine European countries (Estonia, France, Finland, Germany, The Netherlands, Russia, Spain, Sweden, and the United Kingdom) randomised at least one subject.

Independent ethics committees approved the protocol and the trial was conducted in accordance with the Declaration of Helsinki, the International Conference on Harmonisation Good Clinical Practice, and any applicable local regulations. All patients provided written informed consent prior to study initiation. The study is registered with ClinicalTrials.gov (NCT00558363).

\subsection{Randomisation and masking}

Eligible subjects were randomised $1: 1$ to receive dutasteride $0.5 \mathrm{mg}$ or placebo once daily for $2 \mathrm{yr}$. Randomisation to treatment groups was performed in blocks, stratified by previous therapy (RP with or without salvage RT or primary RT) and by centre. GlaxoSmithKline and site personnel, including participants, were masked to study treatment allocation until the study conduct was finished and the database was frozen.

\subsection{Outcome measures}

The primary efficacy assessment was the number of days between start of treatment and the first instance of PSA value being at least twice the latest PSA value before the start of treatment (baseline), followed by confirmation in the immediate, subsequent PSA evaluation when available. Subjects who did not have PSA doubling were censored at the last postbaseline PSA evaluation. PSA evaluations during the study were performed by a central laboratory.

Secondary efficacy assessments included time to disease progression and the proportion of subjects with disease progression, with time to disease progression defined as the number of days between the start of treatment and the earliest of any of the following: PSADT $\leq 3 \mathrm{mo}$, or PSA $>20 \mathrm{ng} / \mathrm{ml}$ (subjects who underwent primary RT) or $>10 \mathrm{ng} / \mathrm{ml}$ (subjects who underwent RP with or without salvage RT) associated with $\geq 50 \%$ increase from baseline PSA and confirmed in an immediate, subsequent PSA determination if available; any biopsy-confirmed progression in the clinical stage ( $T$ stage); need for additional PCa rescue therapy; metastatic disease confirmed by bone scan (bone scans were performed at $24 \mathrm{mo}$, or at the withdrawal visit if applicable, to evaluate the presence of any bone metastases). Subjects who did not have disease progression were censored at the earliest of these dates: the last postbaseline PSA evaluation date; date of last postbaseline bone scan with no metastases; date of last postbaseline ad hoc biopsy with no positive core; last date of successful phone contact during which surgical and nonsurgical intervention were confirmed as none.

Additional secondary efficacy assessments were percentage of subjects with a treatment response (either a PSA decrease or an increase $\leq 15 \%$ from baseline to $\leq 24$ mo of treatment confirmed in all PSA measurements); time to PSA rise from baseline and the proportion of subjects with a PSA rise from baseline, defined as first PSA value showing a $>15 \%$ increase from baseline confirmed in all subsequent measurements; time to PSA progression and percentage of subjects with PSA progression based on the definition of a subject experiencing PSADT $\leq 3 \mathrm{mo}$ or PSA $>20 \mathrm{ng} / \mathrm{ml}$ (subjects who underwent primary RT) or PSA $>10 \mathrm{ng} / \mathrm{ml}$ (subjects who underwent RP with or without salvage RT) associated with $\geq 50 \%$ increase from the baseline PSA measurement and confirmed in an immediate subsequent PSA level determination. Subjects without PSA rise or PSA progression were censored at the last postbaseline PSA evaluation.

Safety assessments included changes on physical examination, adverse events (AEs), vital signs measurements, and laboratory tests. All subjects had PSA level monitored every 3 mo during the treatment phase and then at the follow-up visit ( 4 mo after the end of treatment).

\subsection{Statistical methods}

Given a median time to PSA doubling of 10 to 11 mo for the placebo group [6,7] and assuming $31.8 \%$ of the dutasteride subjects had a PSA doubling at this time point (hazard ratio: 0.605), 110 subjects per treatment arm were required to provide $80 \%$ power to show superiority of dutasteride over placebo using a two-sided log-rank test at $\alpha=0.05$. Assuming a $20 \%$ withdrawal during the study, approximately 138 subjects per treatment arm were needed to be randomised, for a total of 276 subjects.

The primary population for analysis was the intention-to-treat (ITT) population, which included all subjects randomised to study treatment. PSA-related end points considered only the PSA values that might be considered related to the study drug, as restricted by PSA limit date (defined by the latest end-of-treatment visit or laboratory evaluation date and the study drug stop date). PSA evaluations after the PSA limit date were excluded from analysis.

The primary end point was analysed using a log-rank test stratified by previous therapy and investigative-site cluster (defined as a cluster of 
countries based on enrolment). The incidence of PSA doubling was compared using a Mantel-Haenszel test, with the analysis repeated and stratified by previous radical therapy, and was also summarised by subgroups of interest (eg, baseline PSADT $<12 \mathrm{mo}$ and $\geq 12 \mathrm{mo}$ ).

The influence of subject characteristics at study baseline or at first PCa diagnosis on time to PSA doubling was assessed using a Cox proportional hazard regression model. Baseline characteristics considered in the model were age, previous radical therapy, time to screening from radical therapy, baseline PSA, and baseline PSADT. Additional factors considered were last PSA value before radical therapy; Gleason score $(<7$ or $\geq 7)$, $\mathrm{T}$ stage $(\leq \mathrm{T} 1 \mathrm{c}$ or $>\mathrm{T} 1 \mathrm{c}$ ), and risk score (low $=0$, medium $=1$, high $=2$ ) at diagnosis; and hormonal medication use. Duration of previous hormone therapy (HT) for a subject was calculated from the earliest start and latest stop dates of such therapies: when a date was missing, the 15th of the month was imputed, but if month and/or year were missing, the subject was not used in the calculation of duration of HT.

The proportion of subjects with a treatment response was compared between treatment groups using a Mantel-Haenszel test. Other secondary end points were analysed in a similar way to the primary end point.

AEs and changes in clinical laboratory values were evaluated using the ITT population. Other safety measures included gynaecomastia evaluations every 6 mo, and digital rectal examinations and vital signs measurements every $12 \mathrm{mo}$. All summaries were provided by randomised treatment group.

\section{Results}

\subsection{Participants}

Of the 294 subjects randomised (147 in each treatment group), 187 (64\%) completed 24 mo of treatment and 107 discontinued prematurely (71 [48\%] in the placebo group, 36 [24\%] in the dutasteride group) (Fig. 1). The primary reason for discontinuation in both treatment groups was disease progression. Demographics and baseline characteristics were generally similar in the two treatment groups
(Table 1). Fifteen percent of patients (43 of 294) had received previous HT (15\% in the dutasteride group, $14 \%$ in the placebo group). Mean duration of previous HT (from 21 subjects in each treatment group for which this information is available) was $131 \mathrm{~d}$ (146 d in the dutasteride group, $116 \mathrm{~d}$ in the placebo group). Mean time between latest date of HT and screening was $1700 \mathrm{~d}$ (1576 d in the dutasteride group, $1825 \mathrm{~d}$ in the placebo group). The majority of subjects reported being sexually inactive ( $68 \%$ of placebo group, $66 \%$ of dutasteride group) and also impotent (73\% of placebo group, 76\% of dutasteride group) in the 3 mo before screening.

\subsection{Primary end point}

\subsubsection{Overall population}

Dutasteride significantly delayed the time to PSA doubling compared with placebo after $24 \mathrm{mo}$ of treatment $(p<0.001)$. The relative risk $(\mathrm{RR})$ reduction was $66.1 \%$ (95\% confidence interval [CI], 50.35-76.90) for the overall study period. The Kaplan-Meier curves for time to PSA doubling began to diverge from approximately month 6 , and the divergence between the two treatment groups continued to increase through to month 24 (Fig. 2). The incidence of PSA doubling over the 2-yr treatment period (based on ITT subjects with at least one postbaseline PSA evaluation) was $57 \%$ (82 of 144 subjects) in the placebo group and $28 \%$ (41 of 146 subjects) in the dutasteride group ( $p<0.001$ ). The median follow-up time was $722 \mathrm{~d}$ for the dutasteride group and $456 \mathrm{~d}$ for the placebo group (Table 2).

The following baseline variables were associated with time to PSA doubling by Cox proportional hazards regression modelling: type of radical therapy, baseline PSA value, baseline PSADT, and time to screening from radical therapy. Other associated factors were Gleason score and clinical

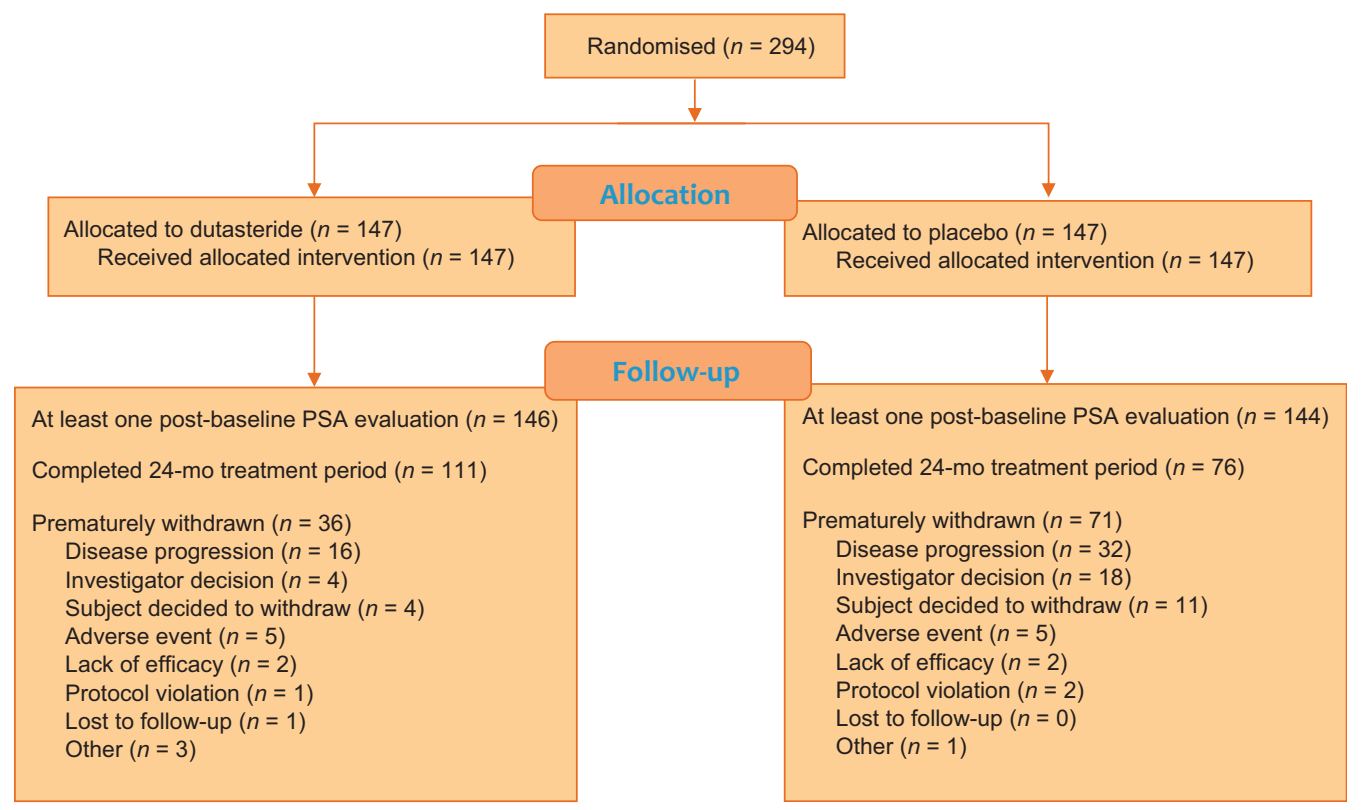

Fig. 1 - Subject disposition. PSA = prostate-specific antigen. 
Table 1 - Subject characteristics at screening/baseline in intention-to-treat population

\begin{tabular}{|c|c|c|}
\hline & Placebo $(n=147)$ & Dutasteride $(n=147)$ \\
\hline \multicolumn{3}{|l|}{ Region (site cluster), no. (\%) } \\
\hline Spain & $57(39)$ & $51(35)$ \\
\hline United Kingdom, The Netherlands, France, Germany & $53(36)$ & $55(37)$ \\
\hline Estonia, Finland, Sweden, Russia & $37(25)$ & $41(28)$ \\
\hline \multicolumn{3}{|l|}{ Previous therapy, no. (\%) } \\
\hline Radical prostatectomy & $119(81)$ & $114(78)$ \\
\hline With salvage therapy & 29 & 23 \\
\hline Without salvage therapy & 90 & 91 \\
\hline Primary radiotherapy & $28(19)$ & $33(22)$ \\
\hline \multicolumn{3}{|l|}{ Sexual function, no. (\%) } \\
\hline Inactive at screening & $100 / 146(68)$ & $97 / 146(66)$ \\
\hline Impotence in the 3 mo before study entry & $106 / 146(73)$ & $110 / 145(76)$ \\
\hline Age, yr, mean (SD, range) & $68.6(6.53,52-81)$ & $69.7(5.76,52-83)$ \\
\hline Race, white, no. (\%) & $146(>99)$ & $147(100)$ \\
\hline Body mass index, $\mathrm{kg} / \mathrm{m}^{2}$, mean ( $\mathrm{SD}$, range) & $27.80(3.761,20.3-42.0)$ & $27.53(3.361,20.9-39.6)$ \\
\hline Baseline PSA <10 ng/mla , no. (\%) & $144(98)$ & $138(94)$ \\
\hline Baseline PSADT $<12 \mathrm{mo}^{\mathrm{a}}$, no. (\%) & $92(63)$ & $88 / 145(61)$ \\
\hline Time to screening from radical therapy $>3 \mathrm{yr}$, no. (\%) & $101(69)$ & $99(67)$ \\
\hline Hormonal medication use before screening no. (\%) & $21(14)$ & $22(15)$ \\
\hline Gleason score $<7$ at diagnosis ${ }^{\mathrm{b}}$, no. (\%) & $73 / 130(56)$ & $78 / 130(60)$ \\
\hline Clinical T stage $>$ T1c at diagnosis ${ }^{\mathrm{b}}$, no. (\%) & $98(67)$ & $97 / 146(66)$ \\
\hline Last PSA value before radical therapy $<10 \mathrm{ng} / \mathrm{ml}^{\mathrm{b}, \mathrm{c}}$, no. (\%) & $82 / 145(57)$ & $84 / 144(58)$ \\
\hline \multicolumn{3}{|l|}{ Risk score, no. (\%) } \\
\hline Low & $34 / 146(23)$ & $48 / 146(33)$ \\
\hline Medium & $77 / 146(53)$ & $65 / 146(45)$ \\
\hline High & $35 / 146$ (24) & $33 / 146(23)$ \\
\hline \multicolumn{3}{|c|}{$\begin{array}{l}\text { SD = standard deviation; PSA = prostate-specific antigen; PSADT = PSA doubling time. } \\
\text { a Latest available value on or before treatment start. } \\
\text { b At time of prostate cancer diagnosis. } \\
\text { c Definitions based on Gleason score at diagnosis }(<7,7 \text {, or }>7) \text {, clinical stage at diagnosis }(<\mathrm{T} 2 \mathrm{a} \text {, T2b-T2c, or }>\text { T2 } \mathrm{c}) \text {, and last PSA before radical therapy }(<10,10- \\
20 \text {, or }>20 \mathrm{ng} / \mathrm{ml}) \text {. Denominators represent the number of data points available when this is not the same as the population. }\end{array}$} \\
\hline
\end{tabular}

tumour stage at diagnosis, and use of HT before screening. Using these variables in the model, the RR reduction in favour of dutasteride increased from $66 \%$ to $70 \%$, supporting a consistent treatment effect. The RR for PSA doubling was higher for subjects with Gleason score $\geq 7$ compared with Gleason score $<7$, and for those with tumour stage $>$ T1c compared with tumour stage $\leq \mathrm{T} 1 \mathrm{c}$. The RR for PSA doubling also increased with higher baseline PSA, lower baseline PSADT, and less time between radical therapy and screening. The RR was lower for primary RT subjects compared with RP subjects and for those who used HT prior to screening compared with those who did not.
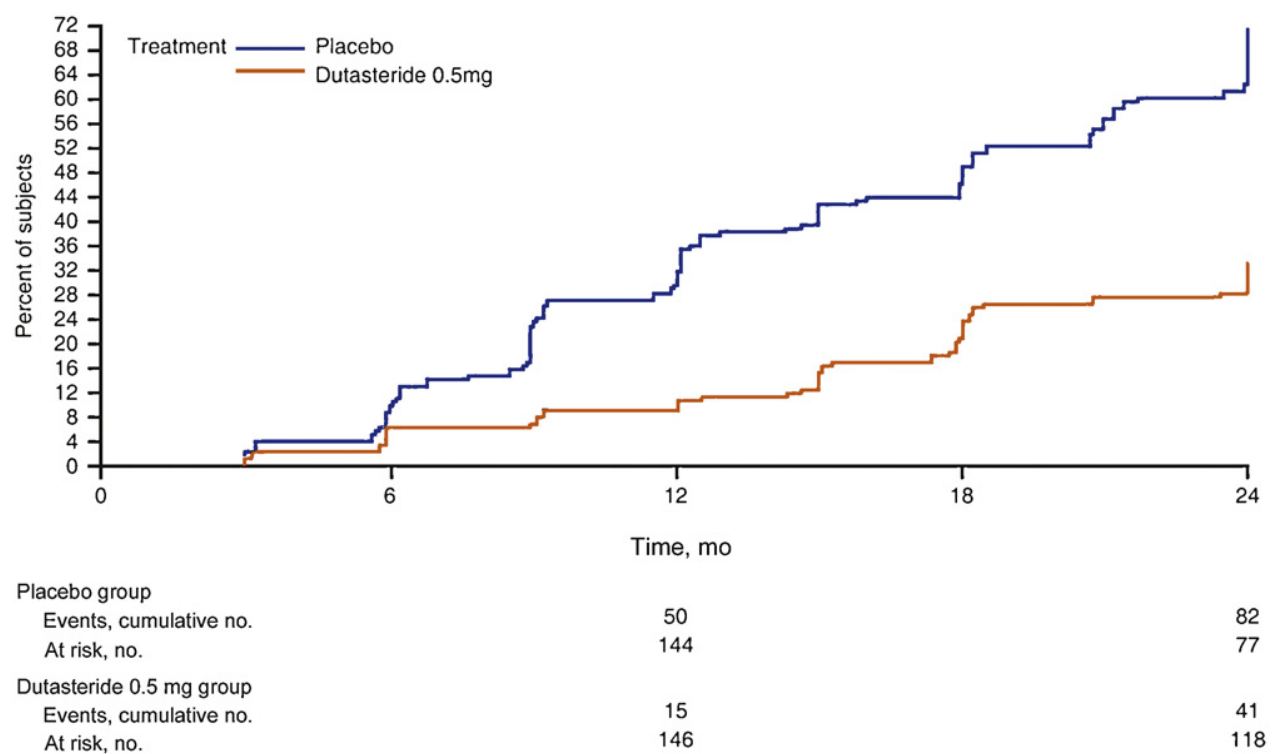

Fig. 2 - Kaplan-Meier estimates of time to prostate-specific antigen doubling (intention-to-treat population). 
Table 2 - Summary of prostate-specific antigen doubling in intention-to-treat population

\begin{tabular}{lll}
\hline & \multicolumn{1}{c}{ Placebo } & Dutasteride \\
\hline Subjects with PSA doubling $^{\mathrm{a}}$, no. (\%) & $82 / 144(57)$ & $41 / 146(28)$ \\
Total person-time $^{\mathrm{b}}, \mathrm{d}$ & 65928 & 86180 \\
Person-time $^{\mathrm{b}}, \mathrm{d}$, median (range) & $456(53-771)$ & $722(22-805)$ \\
Rate of PSA doubling (cases per person-time) & \\
Per day & 0.0012 & 0.0005 \\
Per year & 0.45 & 0.17 \\
\hline
\end{tabular}

PSA = prostate-specific antigen .

${ }^{\text {a }}$ Three placebo subjects and one dutasteride subject did not have a postbaseline PSA measurement and so were not counted in the denominator.

b Follow-up time was the number of days between the randomisedtreatment start date and either PSA-doubling date or censoring date.

Table 3 presents the incidence of PSA doubling by prespecified subgroups. Among those with baseline PSADT $<12 \mathrm{mo}$, the incidence of PSA doubling was 34\% in the dutasteride group and $62 \%$ in the placebo group.

\subsubsection{Response by previous therapy}

The benefit of dutasteride over placebo in delaying the time to PSA doubling was observed both in subjects previously treated with RP ( $p<0.001$; RR reduction: 64.65\%; 95\% CI, 46.64-76.58) and those previously treated with primary RT ( $p=0.007$; RR reduction: 73.94\%; 95\% CI, 25.83-90.84). The incidence of PSA doubling over the 2-yr treatment period was also significantly lower in the dutasteride group than in the placebo group regardless of previous radical therapy (RP: $31 \%$ [35 of 114] vs 59\% [68 of 116], $p<0.001$; primary RT: $19 \%$ [6 of 32 ] vs $50 \%$ [14 of 28 ], $p=0.011$ ).

\subsection{Secondary end points}

Dutasteride significantly delayed disease progression compared with placebo ( $p<0.001$ ); the overall RR reduction in favour of dutasteride was 59\% (95\% CI, 32.53-75.09). The Kaplan-Meier curves for time to disease progression began to diverge from approximately month 6 , and the divergence between the two treatment groups continued to increase through to month 24 (Fig. 3). The incidence of disease progression was $17 \%$ (25 of 146) in the dutasteride group and $34 \%$ (49 of 144 ) in the placebo group ( $p<0.001$ ). The benefit of dutasteride over placebo for reducing the risk of disease progression was observed across PSA-related disease progression events as well as clinical-related outcomes (Table 4). Fewer patients in the dutasteride group required additional rescue therapy and fewer developed bone metastases (nine subjects in the placebo group and four in the dutasteride group had bone metastases; five and two of these cases, respectively, were preceded by another disease progression criterion).

Forty subjects had at least one intervention for PCa during the study ( 29 subjects in the placebo group and 11 in the dutasteride group) (Table 5); only 20 of these were considered as having disease progression (when they were not preceded by another disease progression criterion). There were no surgical interventions, and eight subjects
Table 3 - Incidence of prostate-specific antigen doubling from baseline by subgroups of interest

\begin{tabular}{|c|c|c|}
\hline & $\begin{array}{l}\text { Placebo, } \\
\text { no. }(\%)\end{array}$ & $\begin{array}{l}\text { Dutasteride } \\
0.5 \mathrm{mg} \text {, no. (\%) }\end{array}$ \\
\hline \multicolumn{3}{|l|}{ Subject characteristics at baseline } \\
\hline \multicolumn{3}{|l|}{ Age, yr } \\
\hline$<65$ & $25 / 37(68)$ & $8 / 21(38)$ \\
\hline$\geq 65$ & $57 / 107(53)$ & $33 / 125(26)$ \\
\hline \multicolumn{3}{|l|}{ Site cluster } \\
\hline Spain & $33 / 56(59)$ & $15 / 51(29)$ \\
\hline $\begin{array}{l}\text { United Kingdom, The Netherlands, } \\
\text { France, Germany }\end{array}$ & $32 / 53(60)$ & $16 / 55(29)$ \\
\hline $\begin{array}{l}\text { Scandinavia, Eastern European } \\
\text { Countries }\end{array}$ & $17 / 35(49)$ & $10 / 40(25)$ \\
\hline \multicolumn{3}{|l|}{ Baseline PSA, ng/ml } \\
\hline$<10$ & $82 / 141(58)$ & $37 / 137(27)$ \\
\hline $10-20$ & $0 / 3$ & $4 / 9(44)$ \\
\hline \multicolumn{3}{|l|}{ Baseline PSADT, mo } \\
\hline$<12$ & $55 / 89(62)$ & $30 / 88(34)$ \\
\hline$\geq 12$ & $27 / 55(49)$ & $10 / 56(18)$ \\
\hline \multicolumn{3}{|c|}{ Time from screening to radical therapy, yr } \\
\hline$\leq 1$ & $2 / 3(67)$ & $3 / 3(100)$ \\
\hline$>1$ to $\leq 2$ & $14 / 20(70)$ & $5 / 17(29)$ \\
\hline$>2$ to $\leq 3$ & $17 / 22(77)$ & $19 / 28(68)$ \\
\hline$>3$ & $49 / 99(49)$ & $14 / 98(14)$ \\
\hline \multicolumn{3}{|c|}{$\begin{array}{l}\text { Characteristics of prostate cancer at diagnosis and treatment prior } \\
\text { to baseline }\end{array}$} \\
\hline \multicolumn{3}{|l|}{ Previous therapy } \\
\hline Radical prostatectomy & $68 / 116(59)$ & $35 / 114(31)$ \\
\hline Primary radiotherapy & $14 / 28(50)$ & $6 / 32(19)$ \\
\hline \multicolumn{3}{|l|}{ Gleason score $\mathrm{a}^{\mathrm{a}}$} \\
\hline$<7$ & $33 / 72(46)$ & 19/78 (24) \\
\hline$\geq 7$ & $40 / 57(70)$ & 20/51 (39) \\
\hline \multicolumn{3}{|l|}{ Clinical T stage ${ }^{\mathrm{b}}$} \\
\hline$\leq \mathrm{T} 1 \mathrm{c}$ & $22 / 47(47)$ & $11 / 49(22)$ \\
\hline$>\mathrm{T} 1 \mathrm{c}$ & $60 / 97(62)$ & $30 / 96(31)$ \\
\hline \multicolumn{3}{|c|}{ Last PSA value before radical therapy ${ }^{\mathrm{c}}, \mathrm{ng} / \mathrm{ml}$} \\
\hline$<10$ & $46 / 81(57)$ & $21 / 84(25)$ \\
\hline $10-20$ & $26 / 45(58)$ & $12 / 42(29)$ \\
\hline$>20$ & $10 / 17(59)$ & $6 / 17(35)$ \\
\hline \multicolumn{3}{|l|}{ Hormone therapy used before screening } \\
\hline Yes & $10 / 19(53)$ & $5 / 21(24)$ \\
\hline No & $72 / 125(58)$ & $36 / 125(29)$ \\
\hline \multicolumn{3}{|l|}{ Risk score $^{\mathrm{d}}$} \\
\hline Low & $16 / 33(48)$ & $10 / 48(21)$ \\
\hline Medium & $44 / 76(58)$ & $18 / 65(28)$ \\
\hline High & $22 / 35(63)$ & $13 / 32(41)$ \\
\hline \multicolumn{3}{|c|}{$\begin{array}{l}\text { PSA = prostate-specific antigen; PSADT = prostate-specific antigen } \\
\text { doubling time. } \\
\text { a Gleason score from diagnostic biopsy. } \\
\text { b Clinical tumour stage provided by site as last available staging before } \\
\text { radical therapy. } \\
\text { ' For subjects with radical prostatectomy (with or without salvage } \\
\text { radiotherapy), the latest available PSA value before prostatectomy was used. } \\
\text { For subjects with primary radiotherapy, the latest available PSA value before } \\
\text { the start of radiation therapy was used. } \\
\text { d Definitions are based on Gleason score at diagnosis ( }<7,7 \text {, or }>7) \text {, clinical } \\
\text { stage at diagnosis }(<\mathrm{T} 2 \mathrm{a}, \mathrm{T} 2 \mathrm{~b}-\mathrm{T} 2 \mathrm{c} \text {, or }>\mathrm{T} 2 \mathrm{c}) \text {, and last PSA value before radical } \\
\text { therapy }(<10,10-20 \text {, or }>20 \mathrm{ng} / \mathrm{ml}) \text {. }\end{array}$} \\
\hline
\end{tabular}

received RT (seven in the placebo group, one in the dutasteride group). Most subjects who required rescue therapy received drug treatment ( 22 in the placebo group, 10 in the dutasteride group), usually antiandrogens or luteinising hormone-releasing hormone analogues.

Significantly more patients in the dutasteride group than in the placebo group demonstrated a treatment response at month 12 ( $70 \%$ [ 87 of 124 ] vs $12 \%$ [ 13 of 110 ]; $p<0.001$ ) and month 24 (56\% [62 of 110 ] vs $8 \%$ [ 6 of 76 ]; $p<0.001$ ). 


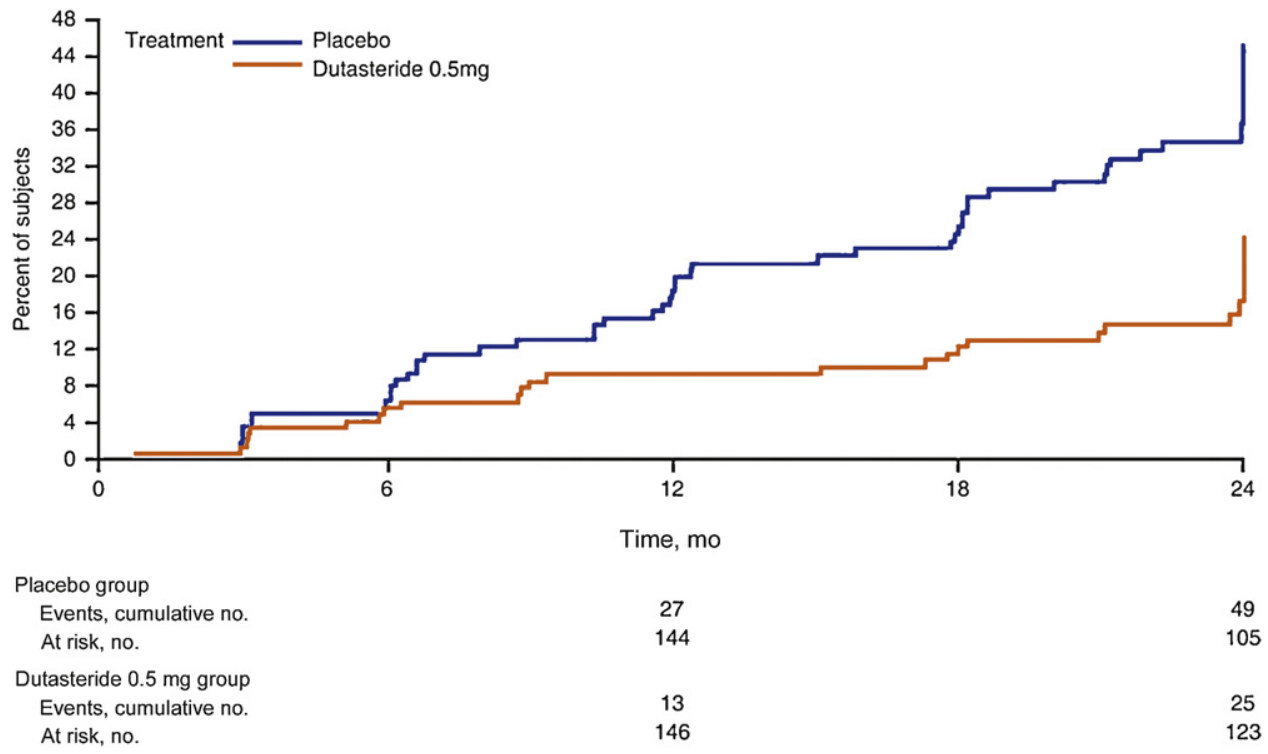

Fig. 3 - Kaplan-Meier estimates of time to disease progression (intention-to-treat population).

The incidence of PSA rise was significantly higher in the placebo group compared with the dutasteride group (88\% [127 of 144 ] vs $49 \%$ [ 72 of 146 ]; $p<0.001$ ). The overall RR reduction estimate in favour of dutasteride was $71.63 \%$ (95\% $\mathrm{CI}, 61.69-79.00$ ). The incidence of PSA progression was numerically higher in the placebo group than in the dutasteride group, although the difference was not statistically significant ( $17 \%$ [ 25 of 144 ] vs $13 \%$ [19 of 146 ]; $p=0.15$ ).

\subsection{Safety assessments}

The incidence of AEs, serious AEs, AEs leading to study discontinuation or withdrawal, and fatal AEs were similar between the treatment groups (Table 6). No fatal AE was

\begin{tabular}{|c|c|c|}
\hline & $\begin{array}{l}\text { Placebo } \\
(n=144)\end{array}$ & $\begin{array}{c}\text { Dutasteride } \\
0.5 \mathrm{mg}(n=146)\end{array}$ \\
\hline Disease progression ${ }^{\mathrm{b}}$, no. (\%) & $49(34)$ & $25(17)$ \\
\hline PSADT $\leq 3$ mo, no. & 7 & 5 \\
\hline Absolute PSA high, no. & 22 & 17 \\
\hline Rescue therapy, no. & 16 & 4 \\
\hline $\begin{array}{l}\text { Clinical progression } \\
\text { (positive biopsy), no. }\end{array}$ & 1 & 0 \\
\hline Bone metastases ${ }^{c}$, no. & 5 & 2 \\
\hline \multicolumn{3}{|c|}{$\begin{array}{l}\text { ITT = intention to treat; PSA = prostate-specific antigen; PSADT = prostate- } \\
\text { specific antigen doubling time. } \\
\text { a Includes ITT subjects who had at least one postbaseline PSA measurement. } \\
\text { b Defined as the first time one of the above disease-progression criteria was } \\
\text { met during the study. If a subject met more than one disease-progression } \\
\text { criterion on the same date, he was counted in each one. } \\
\text { c Nine subjects in the placebo group and four in the dutasteride group had } \\
\text { bone metastases; four and two of these cases, respectively, were preceded by } \\
\text { another disease progression criterion. }\end{array}$} \\
\hline
\end{tabular}

considered to be related to study treatment. The most common AEs (occurring in $\geq 5 \%$ of patients in either treatment group; data not shown) were nasopharyngitis, urinary incontinence, gynaecomastia, hypertension, and back pain.

Breast disorders were more common among subjects in the dutasteride group $(n=10)$ than in the placebo group $(n=4)$. Sexual AEs occurred in few subjects and were less common in the dutasteride group than in the placebo group (Table 6).

Cardiovascular AEs of special interest were reported in four subjects in each treatment group (Table 6). One cardiac failure $\mathrm{AE}$ occurred in the dutasteride group $391 \mathrm{~d}$ after treatment initiation, which was considered by the investigator to be unrelated to study treatment and possibly

Table 5 - Interventions for prostate cancer during the study (intention-to-treat population)

\begin{tabular}{lcc}
\hline Interventions $^{\text {a }}$ & $\begin{array}{c}\text { Placebo, } \\
\text { no. }\end{array}$ & $\begin{array}{c}\text { Dutasteride } \\
0.5 \mathrm{mg}, \text { no. }\end{array}$ \\
\hline Surgical intervention & 0 & 0 \\
Nonsurgical intervention & 29 & 11 \\
First nonsurgical intervention & 22 & 10 \\
$\quad$ Drug therapy & 7 & 1 \\
$\quad$ External beam radiation & 23 & 10 \\
HT used on or after first & 15 & 9 \\
$\quad$ nonsurgical intervention & 0 & 1 \\
Bicalutamide & 2 & 1 \\
Buserelin & 4 & 0 \\
$\quad$ Goserelin & 3 & 3 \\
$\quad$ Flutamide & 8 & \\
Leuprorelin & & \\
$\quad$ Leuprorelin acetate & \multicolumn{2}{l}{} \\
\hline HT = hormone therapy. & & \\
a Some of these interventions were preceded by another criterion of \\
disease progression and so would not have counted as a disease progression \\
criterion for a subject.
\end{tabular}




\begin{tabular}{|c|c|c|}
\hline & $\begin{array}{l}\text { Placebo } \\
(n=147)\end{array}$ & $\begin{array}{c}\text { Dutasteride } \\
0.5 \mathrm{mg}(n=147)\end{array}$ \\
\hline \multicolumn{3}{|l|}{ Adverse events, no. (\%) } \\
\hline Any event & $95(65)$ & $97(66)$ \\
\hline Any serious event & $16(11)$ & $16(11)$ \\
\hline Drug-related event & $15(10)$ & $10(7)$ \\
\hline $\begin{array}{l}\text { Event leading to withdrawal } \\
\text { from the study }\end{array}$ & $5(3)$ & $5(3)$ \\
\hline Fatal event & $2(1)$ & $2(1)$ \\
\hline \multicolumn{3}{|l|}{ Events related to sexual function, no. (\%) } \\
\hline Impotence & $6(4)$ & 0 \\
\hline Altered (decreased) libido & $4(3)$ & $1(1)$ \\
\hline Breast disorders, no. (\%) & $4(3)$ & $10(7)$ \\
\hline \multicolumn{3}{|l|}{ Cardiovascular events, no. (\%) } \\
\hline Any event & $4(2.7)$ & $4(2.7)$ \\
\hline Acute coronary syndrome & $1(0.7)$ & $1(0.7)$ \\
\hline $\begin{array}{l}\text { Ischaemic coronary artery disorders } \\
\text { or atherosclerosis }\end{array}$ & $1(0.7)$ & $1(0.7)$ \\
\hline Ischaemic cerebrovascular events & $1(0.7)$ & $1(0.7)$ \\
\hline Cardiac failure & 0 & $1(0.7)$ \\
\hline Cardiac arrhythmias & $1(0.7)$ & 0 \\
\hline
\end{tabular}

related to a concomitant medication (potassium ascorbate). Dutasteride treatment was continued and the event resolved with specific treatment. In contrast, AEs in the cardiac disorders system organ class (SOC) were more common in the dutasteride group $(n=10)$ than in the placebo group $(n=4)$. No individual AE in this SOC was reported by more than one subject in either treatment group, with the exception of atrial fibrillation (two subjects in the dutasteride group, one subject in the placebo group).

\section{Discussion}

Dutasteride treatment over 2 yr significantly delayed the doubling time for PSA in men with biochemical failure following radical therapy for PCa. Baseline PSA, baseline PSADT, Gleason score and tumour stage at PCa diagnosis, the type of radical therapy received, HT use before screening, and time from radical therapy to screening were all significant predictors of time to PSA doubling. The RR reduction in favour of dutasteride was similar in a model with or without these predictors (70\% and 66\%, respectively), indicating that subjects in this setting may benefit from dutasteride treatment regardless of baseline characteristics. Secondary efficacy end points reinforced the primary efficacy findings. Dutasteride delayed PCa progression, affecting both PSA-related end points and clinical outcomes (need for rescue therapy and the incidence of bone metastases). The observation that bone metastases developed in fewer patients receiving dutasteride than placebo is of interest; however, the actual number of patients affected was small, and a properly powered, prospective study is needed to confirm this finding.

Subgroup analyses according to previous therapy were generally supportive of the results for the overall study population. These analyses should, however, be interpreted with caution given the relatively small sample sizes and the lack of adjustment for multiple end points. Results of other subgroup analyses of interest, including baseline
PSADT, were also consistent with those for the overall population.

The primary end point selected for ARTS was time to PSA doubling from start of randomised treatment. Selection of a PSA-related end point is clinically less meaningful than harder end points such as biopsy-confirmed progression in clinical stage or metastases confirmed by bone scan, and may also have resulted in inherent bias in favour of dutasteride given the effect of treatment on PSA levels. Nevertheless, a serial rise in PSA levels after radical therapy typically predates clinically or radiographically detectable metastatic disease by many years [3], and the population with rising PSA levels represents the second-largest group of patients with PCa [8]. Changes in PSADT may represent a more sensitive end point to detect biological activity than traditional PSA response criteria. For example, in men with a rising PSA level after RP, a median PSADT $<10$ mo is the most significant predictor for progression to metastatic disease [3]. Similarly, a short PSADT following RT is predictive of progression to metastatic disease $[9,10]$.

Dutasteride had predictable and manageable side effects in the study population. The incidence of sexual AEs among men treated with dutasteride was lower than might be expected from the previous safety profile of the drug [11]. However, it is consistent with the impaired sexual function among a substantial proportion of the study population.

Treatment options in men with biochemical progression after radical therapy are limited; therefore, a treatment with limited side effects that delays PSA progression and progression to clinical signs and symptoms could be a useful therapeutic option. Previous studies have investigated agents such as exisulind, rosiglitazone, celecoxib, dietary supplements, and adecatumumab [12-16]. While some of these studies reported encouraging results [12,15], no useful therapies have emerged. The only previous randomised study of a $5 \alpha$-reductase inhibitor (5-ARI) after RP compared 12 mo of treatment with finasteride and placebo in 120 men with serum PSA levels of $0.6-10.0 \mathrm{ng} / \mathrm{ml}$, no evidence of skeletal metastasis, and no previous ADT [17]. Finasteride delayed the increase in PSA by approximately 9 mo compared with placebo. There were also fewer recurrences in the finasteride group, although the difference compared with the placebo group was not statistically significant. In the present study, dutasteride (a dual 5-ARI) resulted in reductions in both PSA-related progression events and also clinical-related outcomes. Nevertheless, additional data would be helpful in deciding if 5-ARIs could be considered a treatment option in this setting.

A potential concern about using 5-ARIs to treat PCa is that they may shorten the time to development of disease that is resistant to ADT [18]. Despite 5-ARIs being in clinical use for $>10 \mathrm{yr}$ and the subject of long-term clinical trials, no such association has yet been reported. However, longerterm follow-up is necessary before this concern can be fully disregarded.

A PSADT of $<9$ mo has been proposed as a cut-off point conferring an increased risk of PCa death and reduced overall survival [19-21]. In ARTS, a cut-off of 12 mo was predefined for the efficacy analyses. Among subjects with a 
baseline PSADT of $<12 \mathrm{mo}$, and therefore at theoretically higher risk of PCa-related outcomes, a smaller proportion of the dutasteride group experienced a PSA-doubling event than the placebo group (34\% vs $62 \%$ ). However, based on previous studies [22,23], the relatively short follow-up period of ARTS is insufficient to confirm if the impact of dutasteride treatment on PSADT translates into overall or PCa-specific survival; this represents a limitation of the study.

Another potential limitation of the study is that investigators and patients were not blinded to PSA test results. Knowledge of PSA level could potentially unblind the study and influence investigators' decisions such as timing of rescue therapy. Revealing PSA values was necessary, however, for investigators to manage their patients and also to explore the impact of the disease and treatment on patient-reported health outcomes (data not shown). Despite this potential limitation, results were consistently in favour of dutasteride across end points, including non-PSA variables such as need for rescue therapy or development of bone metastases.

There was a substantial difference in median follow-up time between the two treatment groups (722 d for the dutasteride group vs $456 \mathrm{~d}$ for the placebo group). This is most likely due to the higher withdrawal rate in the placebo group than in the dutasteride group, which, in turn, was driven by withdrawal due to disease progression and investigator/patient decision to withdraw. Withdrawal due to disease progression was based on objective, predefined criteria, indicating a benefit of dutasteride treatment over placebo. Investigator or patient decisions to withdraw may have been more subjective, and potentially influenced by the PSA unblinding.

\section{Conclusions}

Dutasteride delayed the biochemical progression of PCa in patients with biochemical failure after radical therapy for clinically localised disease. The safety and tolerability profile of dutasteride in this patient population was generally consistent with previous experience with no new safety signals identified. A larger-scale study with a longer followup period is needed to determine whether delayed biochemical progression translates into improved survival.

Author contributions: Fritz Schröder had full access to all the data in the study and takes responsibility for the integrity of the data and the accuracy of the data analysis.

Study concept and design: Schröder, Bangma, Alcaraz, McNicholas, Castro. Acquisition of data: Schröder, Bangma, Angulo, Alcaraz, Colombel, McNicholas, Tammela, Nandy, Castro.

Analysis and interpretation of data: Schröder, Bangma, Angulo, Alcaraz, Colombel, McNicholas, Tammela, Nandy, Castro.

Drafting of the manuscript: Schröder, Bangma, Angulo, Alcaraz, Colombel, McNicholas, Tammela, Nandy, Castro.

Critical revision of the manuscript for important intellectual content: Schröder, Bangma, Angulo, Alcaraz, Colombel, McNicholas, Tammela, Nandy, Castro.

Statistical analysis: Nandy.

Obtaining funding: Schröder.
Administrative, technical, or material support: None.

Supervision: None.

Other (specify): None.

Financial disclosures: Fritz Schröder certifies that all conflicts of interest, including specific financial interests and relationships and affiliations relevant to the subject matter or materials discussed in the manuscript (eg, employment/affiliation, grants or funding, consultancies, honoraria, stock ownership or options, expert testimony, royalties, or patents filed, received, or pending), are the following: Fritz Schröder has acted as a consultant to GlaxoSmithKline (GSK). Teuvo Tammela has acted as a consultant to Amgen, Astellas, Sanofi-Aventis, and Orion Pharma, and received travel grants from Amgen. Tom McNicholas and Ramiro Castro own stock in GSK. Indrani Nandy and Ramiro Castro are employees of GSK. Marc Colombel, Javier Angulo, and Antonio Alcaraz have no conflicts to declare.

Funding/Support and role of the sponsor: Funding for the study was provided by GlaxoSmithKline. The study sponsor designed the study in collaboration with a group of experts and investigators and coordinated the collection, analysis, and interpretation of the data. All authors had full access to trial data and had final responsibility for the decision to submit for publication.

Acknowledgment statement: We are grateful for the support of all investigators and their patients in the conduct of the study. We thank Johannes Wolff, Francesco Montorsi, Pierre Mongiat-Artus, and Per-Anders Abrahamsson for their contributions to the study design. Medical writing support was provided by Tony Reardon of Spirit Medical Communications Ltd and funded by GSK.

\section{References}

[1] Makarov DV, Trock BJ, Humphreys EB, et al. Updated nomogram to predict pathologic stage of prostate cancer given prostate-specific antigen level, clinical stage, and biopsy Gleason score (Partin tables) based on cases from 2000 to 2005. Urology 2007;69:1095-101.

[2] Heidenreich A, Aus G, Bolla M, et al. EAU guidelines on prostate cancer. Eur Urol 2008;53:68-80.

[3] Pound CR, Partin AW, Eisenberger MA, Chan DW, Pearson JD, Walsh PC. Natural history of progression after PSA elevation following radical prostatectomy. JAMA 1999;281:1591-7.

[4] Schröder FH, Bangma CH, Wolff JM, et al. Can dutasteride delay or prevent the progression of prostate cancer in patients with biochemical failure after radical therapy? Rationale and design of the Avodart After Radical Therapy for Prostate Cancer Study. BJU Int 2009;103:590-6.

[5] Roach III M, Hanks G, Thames Jr H, et al. Defining biochemical failure following radiotherapy with or without hormonal therapy in men with clinically localized prostate cancer: recommendations of the RTOG-ASTRO Phoenix Consensus Conference. Int J Radiat Oncol Biol Phys 2006;65:965-74.

[6] Molitierno J, Evans A, Mohler JL, Wallen E, Moore D, Pruthi RS. Characterization of biochemical recurrence after radical prostatectomy. Urol Int 2006;77:130-4.

[7] Lee WR, Hanks GE, Hanlon A. Increasing prostate-specific antigen profile following definitive radiation therapy for localized prostate cancer: clinical observations. J Clin Oncol 1997;15:230-8.

[8] Scher HI, Eisenberger M, D'Amico AV, et al. Eligibility and outcomes reporting guidelines for clinical trials for patients in the state of rising prostate-specific antigen: recommendations from the ProstateSpecific Antigen Working Group. J Clin Oncol 2004;22:537-56.

[9] Patel A, Dorey F, Franklin J, deKernion JB. Recurrence patterns after radical retropubic prostatectomy: clinical usefulness of prostate specific antigen doubling times and log slope prostate specific antigen. J Urol 1997;158:1441-5. 
[10] Zagars GK, Pollack A. Kinetics of serum prostate-specific antigen after external beam radiation for clinically localized prostate cancer. Radiother Oncol 1997;44:213-21.

[11] Evans HC, Goa KL. Dutasteride. Drugs Aging 2003;20:905-16.

[12] Goluboff ET, Prager D, Rukstalis D, et al., UCLA Oncology Research Network. Safety and efficacy of exisulind for treatment of recurrent prostate cancer after radical prostatectomy. J Urol 2001;166:882-6.

[13] Smith MR, Manola J, Kaufman DS, et al. Rosiglitazone versus placebo for men with prostate carcinoma and a rising serum prostate-specific antigen level after radical prostatectomy and/or radiation therapy. Cancer 2004;101:1569-74.

[14] Smith MR, Manola J, Kaufman DS, Oh WK, Bubley GK, Kantoff PW. Celecoxib versus placebo for men with prostate cancer and a rising serum prostate-specific antigen after radical prostatectomy and/or radiation therapy. J Clin Oncol 2006;24:2723-8.

[15] Schröder FH, Roobol MJ, Boevé ER, et al. Randomized, double-blind, placebo-controlled crossover study in men with prostate cancer and rising PSA: effectiveness of a dietary supplement. Eur Urol 2005;48:922-31.

[16] Marschner N, Rüttinger D, Zugmaier G, et al. Phase II study of the human anti-epithelial cell adhesion molecule antibody adecatumumab in prostate cancer patients with increasing serum levels of prostate-specific antigen after radical prostatectomy. Urol Int 2010;85:386-95.
[17] Andriole G, Lieber M, Smith J, et al. Treatment with finasteride following radical prostatectomy for prostate cancer. Urology 1995;45:491-7.

[18] Montorsi F, Alcaraz A, Desgrandchamps F, Hammerer P, Schröder F, Castro F. A broader role for 5ARIs in prostate disease? Existing evidence and emerging benefits. Prostate 2009;69:895-907.

[19] Albertsen PC, Hanley JA, Penson DF, Fine J. Validation of increasing prostate-specific antigen as a predictor of prostate cancer death after treatment of localized prostate cancer with surgery or radiation. J Urol 2004;171:2221-5.

[20] D'Amico AV, Moul JW, Carroll PR, Sun L, Lubeck D, Chen MH. Surrogate end point for prostate cancer-specific mortality after radical prostatectomy or radiation therapy. J Natl Cancer Inst 2003;95:1376-83.

[21] Teeter AE, Presti Jr JC, Aronson WJ, et al. Does PSADT after radical prostatectomy correlate with overall survival? A report from the SEARCH database group. Urology 2011;77:149-53.

[22] Freedland SJ, Humphreys EB, Mangold LA, et al. Risk of prostate cancer-specific mortality following biochemical recurrence after radical prostatectomy. JAMA 2005;294:433-9.

[23] Freedland SJ, Humphreys EB, Mangold LA, et al. Death in patients with recurrent prostate cancer after radical prostatectomy: prostatespecific antigen doubling time subgroups and their associated contributions to all-cause mortality. J Clin Oncol 2007;25:1765-71.

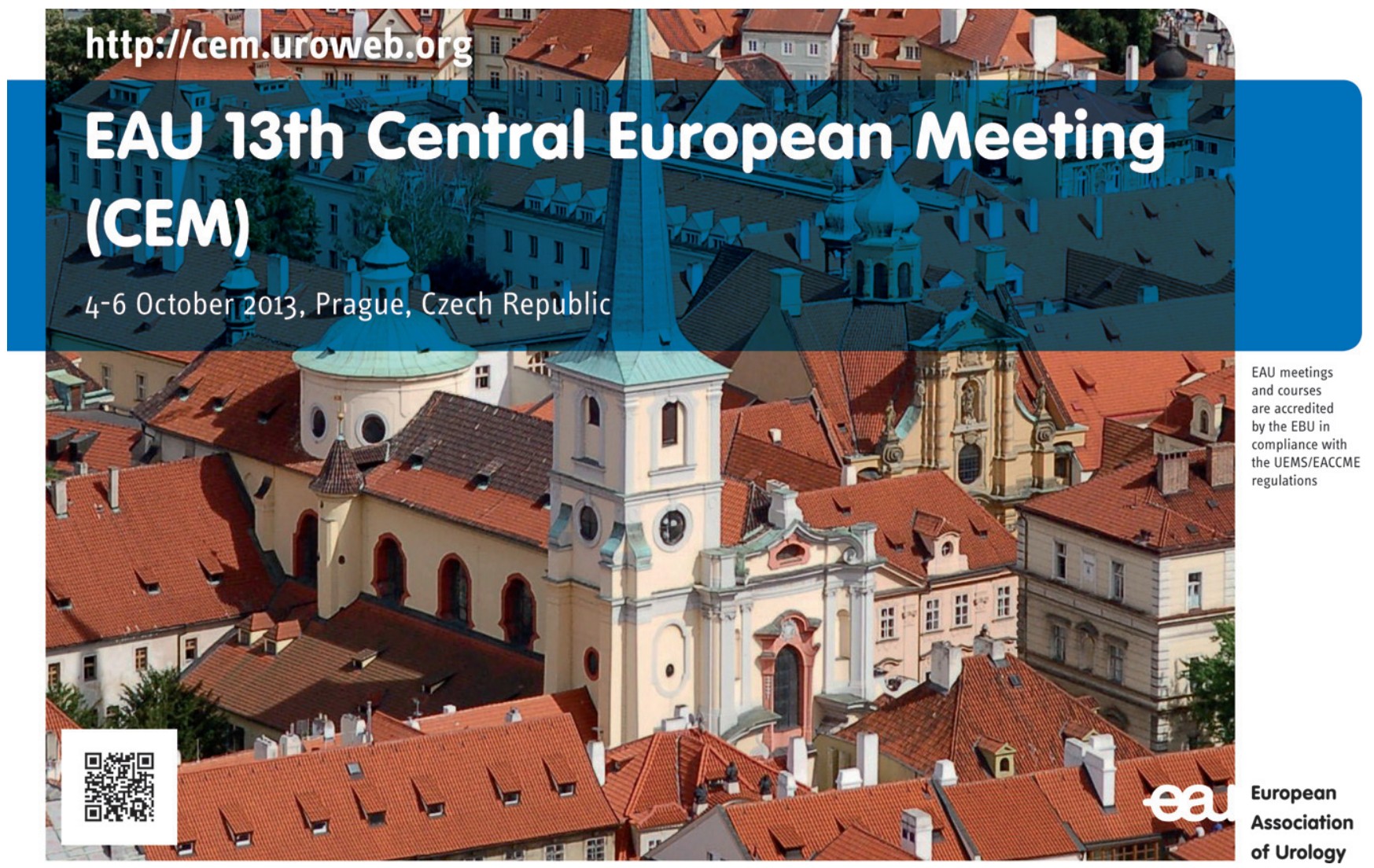

